\title{
Dynamic Crossover in Supercooled Confined Water: Understanding Bulk Properties through Confinement
}

\author{
P. Gallo, ${ }^{*}{ }^{\dagger}$ M. Rovere, ${ }^{\dagger}$ and S.-H. Chen ${ }^{\dagger}$
}

†Dipartimento di Fisica, Università “Roma Tre”, Via della Vasca Navale 84, 00146 Roma, Italy, and ${ }^{\ddagger}$ Department of Nuclear Science and Engineering, Massachusetts Institute of Technology, Cambridge, Massachusetts 02139

\begin{abstract}
We present here molecular dynamics simulations of deeply supercooled SPC/E water confined in a cylindrical pore of MCM-41 silica material. By a layer analysis of the tag particle density correlators, we are able to extract the $\alpha$-relaxation time of the mobile portion of the confined water. This relaxation time is the same as what can be extracted from neutron scattering experiments. From examination of the temperature-dependent behavior of the relaxation time and the dynamic susceptibility, we locate a dynamic crossover at $T=(215 \pm 5) \mathrm{K}$ and a corresponding peak in the specific heat, in agreement with experimental findings in confined water and simulations of the bulk water. Our study demonstrates that the recent results of the experiments on confined water are extremely relevant for the comprehension of low-temperature bulk properties of water.
\end{abstract}

SECTION Statistical Mechanics, Thermodynamics, Medium Effects

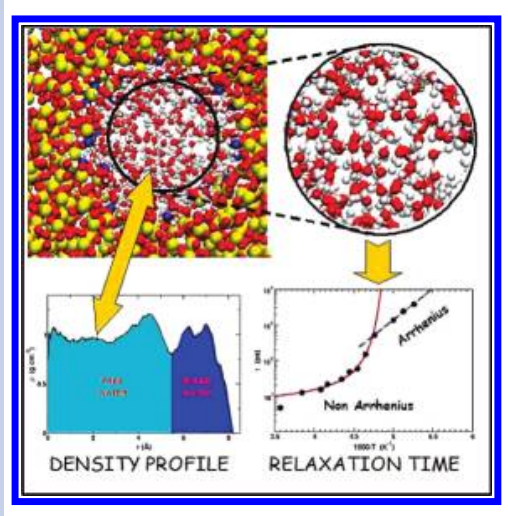

$\mathrm{D}$ espite the considerable progress in the comprehension of the properties of water, a number of anomalies in its behavior remain to be explained, ${ }^{1,2}$ especially in the supercooled region. Thermodynamical response functions, like isothermal compressibility and specific heat at constant pressure, increase anomalously when water is supercooled. ${ }^{3}$ A number of computer simulations and theoretical studies on water support the hypothesis that the anomalous thermodynamical behavior of water is due to the existence of a second critical point ${ }^{4}$ experimentally suggested at around $220 \mathrm{~K}$ and $100 \mathrm{MPa} .{ }^{5}$ This critical point would be the end point of a coexistence line between a low-density liquid (LDL) and a high-density liquid (HDL). The origin of these anomalies might be alternatively related to local density fluctuations that would give rise to a singularity free scenario ${ }^{6}$ or to a critical point free scenario that would allow a first-order liquid-liquid transition without a critical point. ${ }^{7}$

Transport properties, such as shear viscosity and the inverse of the self-diffusion coefficient, also show power law divergences in the supercooled regime toward the singular temperature, ${ }^{8}$ indicating a possible connection between thermodynamics and dynamics for the case of water.

The dynamical behavior of the bulk water simulated upon supercooling ${ }^{9}$ fits in the framework of the idealized version of mode coupling theory (MCT) ${ }^{10}$ When a simple liquid approaches the crossover temperature $T_{\mathrm{C}}$, MCT predicts that the dynamics is dominated by the cage effect.

Liquids which follow the MCT show a relaxation time with a super-Arrhenius behavior. This behavior can be phenomenologically fitted with a Vogel-Fulcher-Tamman (VFT) relation ${ }^{11}$ formula, and these liquids are classified as fragile. Below $T_{\mathrm{C}}$, according to the idealized version of MCT, the system is frozen. In real structural glasses, hopping processes restore ergodicity, and the liquid turns its behavior to that of a strong liquid. ${ }^{10,12}$ The relaxation time of strong liquids increases upon decreasing temperature with an Arrhenius behavior. ${ }^{13}$

Experimental observations demonstrated that bulk water behaves as a fragile liquid, ${ }^{2,8}$ and relaxation times follow MCT predictions ${ }^{14}$ in the temperature range where measurements can be performed. It is important to stress that in water, a network-forming liquid, the caging phenomenon is due to the breaking and re-forming of the hydrogen bond local network. ${ }^{15}$

Experimental investigation of water in the deep supercooled region is hampered by the homogeneous nucleation toward the crystalline phase taking place at ambient pressure in bulk water at $T_{\mathrm{H}}=235 \mathrm{~K}$, although a reversible thermodynamic path appears possible. ${ }^{16}$ For this reason, several experiments are performed on supercooled water in confinement to avoid crystallization. Quasielastic neutron scattering (QENS) experiments performed on supercooled water in confinement ${ }^{17,18}$ found evidence of a fragile to strong (FS) dynamic crossover already hypothesized in bulk water, ${ }^{19,20}$ in analogy with other network-forming liquids. ${ }^{12}$

Recently a possible relation between the presence of a liquid-liquid transition in bulk water and the FS dynamical crossover has been shown. ${ }^{18,21-23}$ Upon getting closer to the critical point, the lines of maxima of different response functions tend to converge to the so-called Widom line. ${ }^{24,25}$ The Widom line separates the water with HDL-like feature at high temperatures from that with LDL-like features at low temperatures. The maximum found in the specific heat at the

Received Date: November 25, 2009

Accepted Date: January 14, 2010 


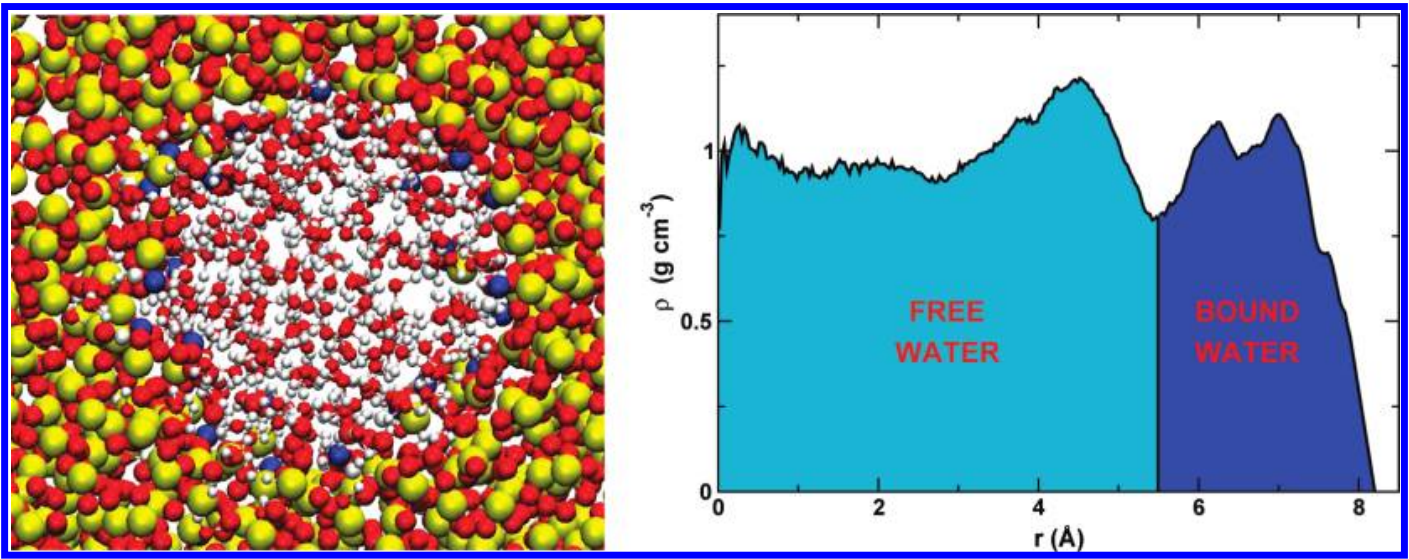

Figure 1. Left: projection in the $x y$ plane of the snapshot of the $N=380$ water molecules configuration inside of the silica cavity at $T=220 \mathrm{~K}$. A portion of the silica surface is shown. The larger spheres are silicon atoms, and the darker are acidic hydrogens. Right: density profile of oxygen atoms of water along the pore at $T=300 \mathrm{~K}$. With the chosen number of particles, the effective density inside of the pore is around $0.98 \mathrm{~g} / \mathrm{cm}^{3}$, while the density in the inner part of the pore $(0<r<5.5 \AA)$ is around $1.0 \mathrm{~g} / \mathrm{cm}^{3}$.

FS crossover has been related in the literature both in simulation on bulk water ${ }^{21}$ and in neutron scattering experiments in confinement ${ }^{17}$ to the crossing of the Widom line. Therefore, in water, the Widom line would be also a switching line for hopping, favored on the side where water is less dense.

It appears crucial to assess to what extent the properties of water in confinement are related to the bulk. We present in this Letter evidence from molecular dynamics (MD) simulations that a FS crossover similar to that in the bulk takes place in supercooled confined water. We will show that under a proper analysis of the density correlators, able to extract from dynamics the same features measured in QENS, we recover a bulk-like behavior. In this way, we can connect MD results in bulk and experiments in confined water.

We simulated water in an MCM-41 pore, where the FS crossover was first measured. ${ }^{17}$ The pore was modeled to catch the main features of the pores of MCM-41 silica material.

In a cubic cell with a box length of $42.78 \AA$, silica atoms at a density of $0.0662 \AA^{-3}$ were simulated with the Vessal et al. potential. ${ }^{26}$ The system initially in the $\beta$-cristobalite crystalline structure was melted at $6000 \mathrm{~K}$ and then driven and equilibrated toward $1000 \mathrm{~K}$. After that, the melted silica was quenched at a cooling rate of $10^{13} \mathrm{~K} / \mathrm{s}$ to $300 \mathrm{~K}$ to obtain a glassy phase. Inside of the cubic cell of silica glass, we carved a cylindrical pore of $15 \AA$ in diameter. This radius was chosen to make contact with experiments. ${ }^{17,18}$ The cavity was cut out by eliminating all of the atoms lying within a distance of $7.5 \AA$ of the central axis of the cube, taken as the $z$-axis. After relaxing the cavity for a few picoseconds, we eliminated all of the silicon atoms with less than four oxygen neighbors. After this procedure, in the system, there were bridging oxygens (BO) with two silicon neighbors and nonbridging oxygens (NBO) with only one silicon neighbor. The dangling bonds of the NBO were saturated with hydrogen ions, acidic hydrogens (AHs). With this procedure, we obtained a cavity with a roughly cylindrical shape and a corrugated surface. During the simulation, all of the atoms of the confining matrix were kept rigid.

We inserted in the pore $N=380$ water molecules. The number was tuned to get a density of around $1 \mathrm{~g} / \mathrm{cm}^{3}$ in the
Table 1. Bridging Oxygens (BO), Nonbridging Oxygens (NBO), Silicons (Si), and Acidic Hydrogens (AH) Potential Parameters ${ }^{29}$

\begin{tabular}{lccc}
\hline & $q(e)$ & $\sigma(\AA)$ & $\varepsilon(K)$ \\
\hline $\mathrm{BO}$ & -0.629 & 2.70 & 230 \\
$\mathrm{NBO}$ & -0.533 & 3.00 & 230 \\
$\mathrm{Si}$ & 1.283 & - & - \\
$\mathrm{AH}$ & 0.206 & - & -
\end{tabular}

inner part of the pore. The water molecules were described by the SPC/E model. ${ }^{27}$ The SHAKE algorithm was employed to integrate the equations of motion. Water molecules interact with the atoms of the matrix by means of a silica-water force field defined by the parameters reported in Table 1 . We used the Lorentz-Berthelot mixing rules for the Lennard-Jones part of the interaction. Since the simulations of such a system are time-consuming, we used the shifted force method with a cutoff at $9 \AA$ for all interactions. We checked that the use of a larger cutoff or Ewald summations did not change the trend of the obtained results; see also ref 28. Periodic boundary conditions were applied. The molecular dynamics was performed in the microcanonical ensemble with a time step of $1 \mathrm{fs}$. Each run was equilibrated via a coupling to a temperature reservoir by using the Berendsen method of velocity rescaling. We simulated temperatures ranging from $T=300$ to 190 K. For each temperature, equilibration runs between $5 \mathrm{~ns}$ at high $T$ and 12 ns at low $T$ were performed. The self-intermediate scattering function (SISF) was calculated from a number of equilibrated configurations ranging from $10 \mathrm{~ns}$ at high $T$ to $20 \mathrm{~ns}$ at low $T$. The total time required for the simulations presented in this work was about $20000 \mathrm{~h}$ ( $\sim 2.5$ years on a single processor). Lengthly simulations are required because already at ambient temperature, the SISF decays on a very long time scale.

We report in Figure 1 a a snapshot of the water configuration after equilibration. A number of water molecules are attracted to the silica surface and form hydrogen bonds with the oxygens or the AHs of the surface. Strong inhomogeneities are present in the system, and in the density profile of water, a distinct layer close to the hydrophilic surface is visible, as shown in Figure $1 \mathrm{~b}$. 


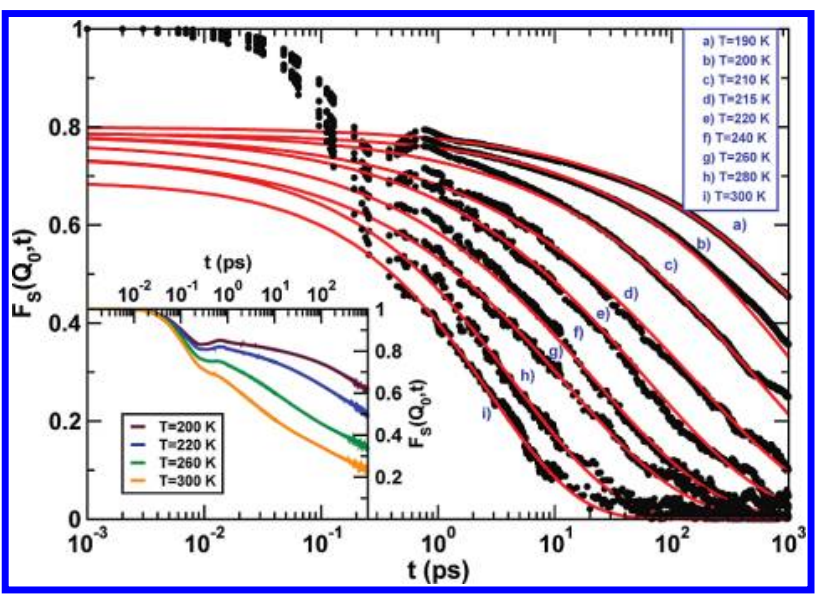

Figure 2. Main frame: self-intermediate scattering function (SISF) of the oxygen atoms of the free water for $Q_{0}=2.25 \AA^{-1}$ from $T=$ 300 (bottom) to $190 \mathrm{~K}$ (top). The continuous lines are the fit to the Kohlrausch-William-Watts $(\mathrm{KWW})$ formula $F_{\mathrm{s}}(Q, t) \rightarrow f_{\mathrm{O}} \mathrm{e}^{-(t / \tau)^{\rho}}$, where $f_{\mathrm{Q}}$ is the height of the plateau, $\tau$ is the $\alpha$-relaxation time, and $\beta$ is the Kohlrausch exponent. Inset: SISF of the oxygen atoms of all of the water molecules for $Q_{0}=2.25 \AA^{-1}$ for a selected set of temperatures.

The SISF $F_{s}(Q, t)$ is the space Fourier transform of the tag particle density-density correlation function. From the analysis of this correlator, we can extract the $\alpha$-relaxation time, that is, the slow relaxation time that liquids develop upon supercooling. We show in Figure 2 the SISF of the oxygen atom of water molecules at the peak of the oxygen-oxygen structure factor. The SISF calculated for all of the water molecules, total SISF, is shown in the inset. Since the total correlators show a long tail extending well beyond the $10 \mathrm{~ns}$ time scale already at ambient temperature, there are water molecules in the pore that are, on average, extremely slow at all temperatures. Besides, the shape of the total correlation function strongly deviates from the expected behavior for supercooled liquids, which is either an exponential at highest temperatures (Brownian relaxation) or a stretched exponential at lower temperatures ( $\alpha$-relaxation). These deviations are caused by the highly hydrophilic nature of the substrate. The dynamics of the extremely slow water molecules close to the surface of the pore superposes to the behavior of the rest of the water. Similar to water in Vycor, ${ }^{29}$ a layer analysis of the density correlators must be performed in order to recover a bulk-like behavior. In the present case, we found that we recover a stretched exponential decay if we consider only the dynamics of water contained in the layer defined by a radius 0 $<r<5.5 \AA$, where $r^{2}=x^{2}+y^{2}$. As shown in Figure $1 \mathrm{~b}$, we define the water in this layer as the free water. This water contains molecules that are far enough from the surface not to be engaged in hydrogen bonds with the substrate. The remaining water molecules in the monolayer close to the strongly hydrophilic surface, bound water, are practically immobile and can be identified by the first distinct layer close to the substrate in the density profile. The percentage of free water in our pore is around $70 \%$.

In the main frame of Figure 2, we show the SISF calculated only for the "free water", partial SISF. It is evident by looking at the partial SISF that the behavior of the correlator is

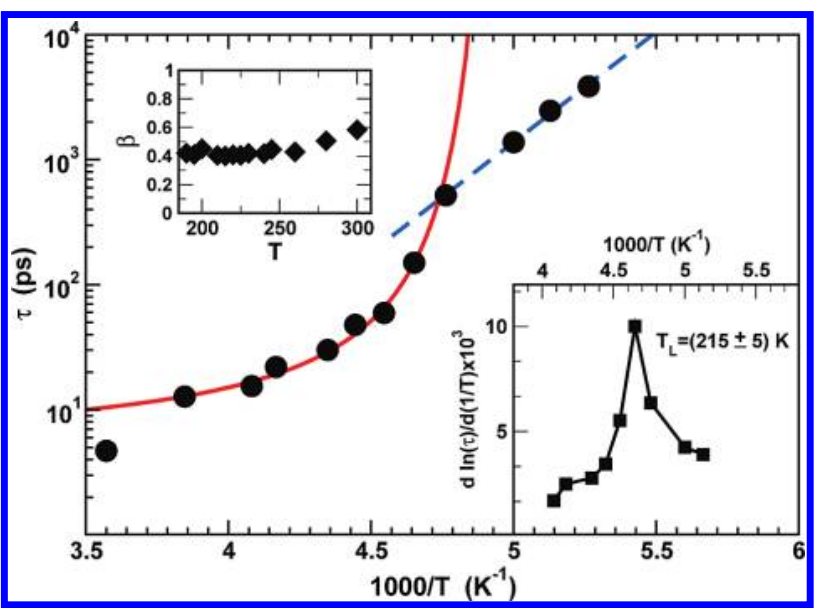

Figure 3. Main frame: Arrhenius plot of the relaxation time $\tau$ as a function of $1000 / T$. At high temperature, below $300 \mathrm{~K}$, the points are fitted with the VFT formula (bold line) $\tau=\tau_{0}^{\mathrm{VFT}} \exp \left[B T_{0} /(T-\right.$ $\left.T_{0}\right)$ ], where $B$ is the fragility parameter and $T_{0}$ is an ideal glass transition temperature located below the experimental glass transition temperature $T_{\mathrm{g}}$. The parameters extracted from the fit are $B=0.25$ and $T_{0}=200 \mathrm{~K}$. At low temperature, the fit is done with the Arrhenius function (long dashed line) $\tau=\tau_{0}^{\mathrm{A}} \exp \left[E_{\mathrm{A}} / k_{\mathrm{B}} T\right]$, where $E_{\mathrm{A}}$ is the activation energy. We find from our data an activation energy of $E_{\mathrm{A}}=34 \mathrm{~kJ} / \mathrm{mol}$. Lower inset: inverse temperature derivative of the logarithm of $\tau$. Upper inset: $\beta$ values

now similar to what was found in the bulk water. ${ }^{9}$ We observe upon supercooling the onset of a plateau region after an initial ballistic regime. After the plateau, it develops a long time decay with the features of an $\alpha$-relaxation that, at variance with the total SISF, can be described by a KohlrauschWilliam-Watts (KWW) function. Except for the lowest temperatures, this correlator decays to 0 between 100 and 1000 ps. The analysis of the free water is therefore particularly relevant since in QENS experiments, only mobile water contributes to the measured correlation functions. In fact, due to the instrumental energy resolution function, the dynamics that happens on time scales larger than 10 ns is buried inside of the line shape of the resolution and is therefore nonmeasurable in neutron scattering experiments.

A fitting procedure of the free water SISF can be performed in terms of the KWW formulas. The fits are also reported in Figure 2.

The relaxation times obtained from the fit are reported in the main frame of Figure 3. We see that by decreasing the temperature below $280 \mathrm{~K}$, the system enters into a regime where the relaxation time has a fragile behavior. In analogy with experiments on MCM-41, we fitted it with the VFT relation. Below $T=210 \mathrm{~K}$, the relaxation time shows a crossover to the Arrhenius $T$ dependence. In order to precisely locate the temperature of the crossover, we calculated the inverse temperature derivative of $\ln (\tau)$. This quantity is shown in the lower inset of Figure 3, where we see that a very sharp peak is present, from which we can estimate the crossover temperature as $T_{\mathrm{L}}=(215 \pm 5) \mathrm{K}$. In the upper inset, we report the values of the stretching parameter $\beta$ obtained from the fit. The values of the $\beta$ exponent in confinement appear lower than the bulk ${ }^{9}$ but with a similar trend. Compatible values have been obtained from QENS experiments on confined water in MCM-41. ${ }^{17}$ 


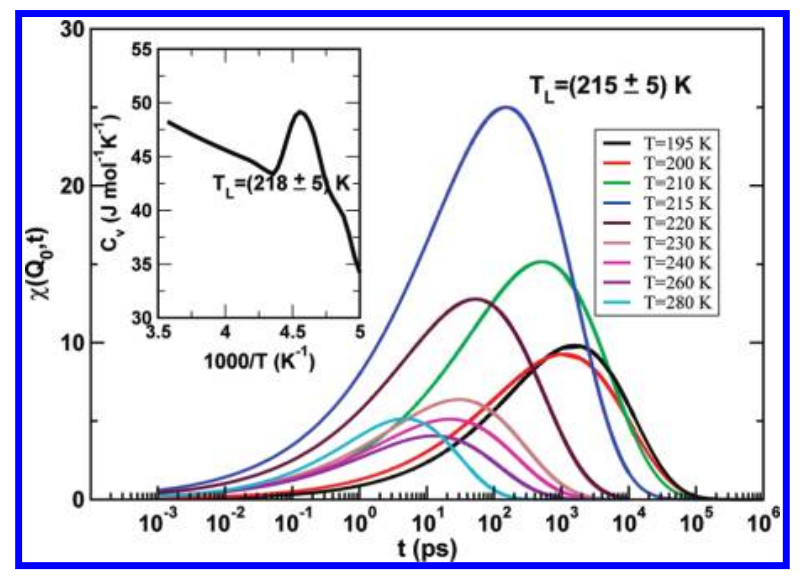

Figure 4. Main frame: dynamic susceptibilities calculated for all of the temperatures investigated with the formula ${ }_{2} \chi_{\mathrm{T}}(Q, t)=$ $-\left[\delta F_{\mathrm{S}}(Q, t) / \delta T\right]=F_{\mathrm{S}}(Q, t) \beta\left(t / \tau(Q, t)^{\beta}[\delta \ln \tau(Q, t) / \delta(1 / T)] T^{-2}\right.$. The temperature of the highest curve corresponds to $T_{\mathrm{L}}$. Inset: specific heat of the system as function of $1000 / T$.

A recent QENS experimental study of the dynamical susceptibility $\chi_{\mathrm{T}}(Q, t)$ on confined water has shown that this quantity can be used to locate $T_{\mathrm{L}}{ }^{30}$ The peak height of $\chi_{\mathrm{T}}(Q, t)$ grows as $T$ is lowered and reaches a maximum when the system undergoes the dynamic crossover. We report this quantity calculated from our data in the main frame of Figure 4 . A high peak is visible for the $\chi_{\mathrm{T}}(Q, t)$ corresponding to $T=215 \mathrm{~K}$.

In order to verify the relation between the Widom line and the dynamic crossover, we calculated the specific heat of the system for all of the temperatures investigated. The result, obtained by computing the derivative of the internal energy with respect to temperature, is reported in the inset of Figure 4. We observe a well-defined peak at $T=(218 \pm 5) \mathrm{K}$, consistent with the value of $T_{\mathrm{L}}$ obtained from the behavior of $\tau$ and $\chi_{\mathrm{T}}(Q, t)$.

The FS crossover in confined SPC/E water found here can also be compared with the result for bulk SPC/E, where it has been located at around $T=210 \mathrm{~K}$ for the $\rho=1 \mathrm{~g} / \mathrm{cm}^{3}$ isochore. $^{31}$

We have shown in this Letter that MD simulations of bulk and confined water describe the same phenomenology upon supercooling as far as the cooperative properties are concerned, provided a layer analysis is performed in the confined system. In our confined system, the temperature of the FS crossover obtained from the relaxation time in the "free water" is consistent with the crossover transition found in the dynamical response function and with the maximum of the specific heat.

We believe that the findings reported in this Letter represent a big step forward in the comprehension of the water anomalous behavior in the supercooled region. Our Letter in fact bridges the gap present between experiments in confinement; see, for example, refs 17 and 18 and MD results in the bulk, $^{21}$ showing for the first time that the results of the numerous neutron scattering experiments that were done in confinement are valid also for the bulk phase.

\section{AUTHOR INFORMATION}

\section{Corresponding Author:}

*To whom correspondence should be addressed. E-mail: gallop@ fis.uniroma3.it.
ACKNOWLEDGMENT The research at MIT is supported by DOE Grant DE-FG02-90ER45429. P.G. and M.R. gratefully acknowledge the computational support of the Roma Tre INFN-GRID

\section{REFERENCES}

(1) Debenedetti, P. G.; Stanley, H. E. Supercooled and Glassy Water. Phvs. Todav 2003, 56, 40-46

(2) Debenedetti, P. G. Supercooled and Glassy Water. I. Phys.: Condens. Matter 2003, 15, R1669-R1726.

(3) Speedy, R. J.; Angell, C. A. Isothermal Compressibility of Supercooled Water and Evidence for a Thermodynamic Singularity at $-45^{\circ}$ C. L. Chem. Phys. 1976, 65, 851-858.

(4) (a) Poole, P. H.; Sciortino, F.; Essman, U.; Stanley, H. E. Phase Behavior of Metastable Water. Nature 1992, 360, 324-328. (b) Poole, P. H.; Sciortino, F.; Essman, U.; Stanley, H. E. Spinodal of Liquid Water. Phys. Rev. E 1993, 48, 3799-3817.

(5) (a) Mishima, O.; Stanley, H. E. Decompression-Induced Melting of Ice IV and the Liquid-Liquid Transition in Water. Nature 1998, 392, 164-168. (b) Mishima, O.; Stanley, H. E. The Relationship between Liquid, Supercooled and Glassv Water. Nature 1998, 396, 329-335

(6) Sastry, S.; Debenedetti, P. G.; Sciortino, F.; Stanley, H. E. Singularity-Free Interpretation of the Thermodynamics of Supercooled Water. Phvs. Rev. E 1996, 53, 6144-6154.

(7) Angell, C. A. Insights into Liquid Water Phases from Study of its Unusual Glass-Forming Properties. Science 2008, 319 , 582-587.

(8) Prielmeier, F. X.; Lang, E. W.; Speedy, R. J.; Lüdemann, H.-D. Diffusion in Supercooled Water to $300 \mathrm{MPa}$. Phys. Rev. Lett. 1987, 59, 1128-1131

(9) Gallo, P.; Sciortino, F.; Tartaglia, P.; Chen, S.-H. Slow Dynamics of Water Molecules in Supercooled States. Phvs. Rev. Lett. 1996, 76, 2730-2733

(10) Götze, W. Complex Dynamics of Glass-Forming Liquids: A Mode-Coupling Theory; Oxford University Press: Oxford, U. K., 2009

(11) Angell, C. A. Formation of Glasses from Liquids and Biopolymers. Science 1995, 267, 1924-1935 (1995).

(12) Saika-Voivod, I.; Poole, P. H.; Sciortino, F. Fragile-to-Strong Transition and Polyamorphysm in the Energy Landscape of Liquid Silica. Nature 2001, 412, 514.

(13) Martinez, L. M.; Angell, C. A. A Thermodynamic Connection to the Fragility of Glass-Forming Liquids. Nature 2001, 410, 663-667.

(14) Torre, R.; Bartolini, P.; Righini, R. Structural Relaxation in Supercooled Water by Time-Resolved Spectroscopy. Nature 2004, 428, 296-299

(15) Starr, F. W.; Nielsen; Stanley, H. E. Fast and Slow Dynamics of Hudrogen Bonds in Liquid Water. Phvs. Rev. Lett. 1999, 82, 2294

(16) Speedy, R. J.; Debenedetti, P. G.; Smith, S. R.; Huang, C.; Kay, B. D. The Evaporation Rate, Free Energy, and Entropy of Amorphous Water at $150 \mathrm{~K}$. I. Chem. Phys. 1996, 105, 240-244.

(17) Faraone, A.; Liu, L.; Mou, C.-Y.; Yen, C.-W.; Chen, S.-H. Fragileto-Strong Liquid Transition in Deeply Supercooled Confined Water. I. Chem. Phvs. 2004, 121, 10843-10846

(18) Liu, L.; Chen, S.-H.; Faraone, A.; Yen, C.-W.; Mou, C.-Y. Pressure Dependence of Fragile-to-Strong Transition and a Possible Second Critical Point in Supercooled Confined Water. Phys. Rev. Lett. 2005, 95, 117802.

(19) Ito, K.; Moynihan, C. T.; Angell, C. A. Thermodynamic Determination of Fragility in Liquids and a Fragile-to-Strong Liquid Transition in Water. Nature 1999, 398, 492-495. 
(20) Angell, C. A. Amorphous Water. Annu. Rev. Phys. Chem. 2004, $55,559-583$

(21) $\mathrm{Xu}, \mathrm{L}$; ; et al. Relation between the Widom Line and the Dynamic Crossover in Systems with a Liquid-Liquid Phase Transition. Proc. Natl. Acad. Sci. U.S.A. 2005, 102, 1655816562.

(22) Mallamace, F; et al. NMR Evidence of a Sharp Change in a Measure of Local Order in Deeply Supercooled Confined Water. Proc. Natl. Acad. Sci. U.S.A. 2008, 105, 12725-12729.

(23) Yoshida, K.; Yamaguchi, T.; Kittaka, S.; Bellissent-Funel, M.-C.; Fouquet, P. Thermodynamic, Structural, and Dynamic Properties of Supercooled Water Confined in Mesoporous MCM-41 Studied with Calorimetric, Neutron Diffraction, and Neutron Spin Echo Measurements. L. Chem. Phys. 2008, 129, 054702.

(24) Xu, L. M.; et al. Appearance of a Fractional Stokes-Einstein Relation in Water and a Structural Interpretation of its Onset. Nat. Phvs. 2009, 5, 565-569.

(25) Franzese, G.; Stanley, H. E. The Widom Line of Supercooled Water. L.Phvs.: Condens. Matter 2007, 19, 205126.

(26) Vessal, B.; Amini, M.; Catlow, C. R. A. Computer Simulation of the Structure of Silica Glass. I. Non-Cryst. Solids 1993, 159, 184-186.

(27) Berendsen, H. J. C.; Grigera, J. R.; Straatsma, T. P. The Missing Term in Effective Pair Potentials. L.Phvs. Chem. 1987, 91, 6269-6271.

(28) Mark, P.; Nilsson, L. Structure and Dynamics of the TIP3P, SPC, and SPC/E Water Models at 298 K. L.Phvs. Chem A 2001, 105, 9954-9960.

(29) (a) Gallo, P.; Rovere, M.; Spohr, E. Supercooled Confined Water and the Mode Coupling Crossover Temperature. Phvs. Rev. Lett. 2000, 85, 4317-4320. (b) Gallo, P.; Rovere, M.; Spohr, E. Glass Transition and Layering Effects in Confined Water: a Computer Simulation Study. I. Chem. Phys. 2000, 113, 11324-11335.

(30) Zhang, Y.; et al. Dynamic Susceptibility of Supercooled Water and its Relation to the Dynamic Crossover Phenomenon. Phvs. Rev. E 2009, 79, 040201 (R).

(31) Starr, F. W.; Sciortino, F.; Stanley, H. E. Dynamics of Simulated Water under Pressure. Phys. Rev. E 1999, 60, 6757-6768. 